\title{
Appareillage d'épitaxie de composés semiconducteurs par transport réactif à courte distance
}

\author{
J. M. Laroche et G. Cohen-Solal \\ Laboratoire de Physique du Solide, CNRS-Bellevue, 92190 Meudon, France
}

(Reçu le 31 janvier 1983, révisé le 5 avril, accepté le 12 avril 1983)

\begin{abstract}
Résumé. - L'appareillage décrit est un système permettant de réaliser le transport d'un matériau sur un substrat, dans une enceinte où la pression - ou le débit — du gaz réactif peuvent être fixés; les caractéristiques principales résident dans le contrôle et la stabilisation des températures, la rapidité d'établissement des programmes thermiques et la multiplicité des interventions in situ. Précision des mesures et souplesse d'utilisation sont les qualités qui font de cet appareillage un bon outil de recherche expérimentale. Un exemple d'application à la croissance épitaxique d'un composé semiconducteur (CdTe) est présenté.
\end{abstract}

\begin{abstract}
We describe an apparatus which is used for the transport of a material upon a substrate, in a vessel where the pressure or the flow rate of the reactive gas are determined; the mean characteristics are the control and the stability of the temperature, the fast settlement of the thermal program and the possibility of in situ operations. Measurement accuracy and use flexibility make this apparatus well adapted to experimental research. The epitaxial growth of a compound semiconductor (CdTe) is given as an example of application.
\end{abstract}

\section{Introduction.}

La technique de transport réactif, en phase vapeur et à courte distance dite C.S.V.T. (Close-Spaced Vapour Transport) s'est révélée particulièrement efficace pour le dépôt de semiconducteurs tels que le silicium [1] et le germanium [2] et les composés comme GaAs [3, 7], $\mathrm{CdS}[8,9]$ et CdTe [10-14]. Les appareillages utilisés, bien qu'efficaces, sont assez sommaires; ils sont en général constitués par un tube de silice ouvert aux deux bouts, à travers lequel circulent les mélanges de gaz réactifs, avec dans la partie médiane deux blocs de graphite superposés entre lesquels se placent source et substrat séparés par une cale annulaire de silice délimitant une micro-enceinte de réaction. Le chauffage des blocs est assuré par absorption de rayonnement infrarouge émis par deux lampes extérieures et la température lue par les thermocouples associés. Dans ces conditions l'étude des caractéristiques de transport (vitesse de croissance) du processus réactif (énergie d'activation) et des propriétés des couches (cristallinité - composition chimique - propriétés de transport électronique) se heurte à la fois au manque de souplesse dans l'utilisation et au manque de précision dans la détermination des différents paramètres de l'expérience. En outre l'absence de possibilités d'intervention in situ entraîne une certaine limitation, surtout dans le cadre de recherches orientées vers l'obtention de dispositifs. D'où la nécessité de concevoir et de réaliser un appareillage mieux adapté à la recherche avec les caractéristiques suivantes :

- Possibilité d'une programmation thermique.

- Atteinte rapide et stabilisation des températures affichées.

- Détermination précise des paramètres de la croissance entraînant de ce fait une bonne reproductibilité expérimentale.

- Possibilité d'interventions multiples in situ.

Outre la proximité de la source et du substrat (de l'ordre de $1 \mathrm{~mm}$ de distance, fixé par l'épaisseur de la cale de silice), entre lesquels on établit une différence de température, la caractéristique de la méthode réside dans le fait qu'un gaz est utilisé pour réagir sur le matériau source en donnant des composés volatils, qui diffusent jusqu'à la surface du substrat où ils se décomposent en déposant le matériau source et en régénérant le gaz réactif, utilisable pour un nouveau cycle. Dans le cas du transport de composés semiconducteurs tels que $\mathrm{CdS}$, CdTe et GaAs les gaz réactifs généralement utilisés en C.S.V.T. sont l'hydrogène ou la vapeur d'eau. 


\section{Description de l'appareillage.}

L'ensemble du dispositif est constitué par une enceinte à vide, reliée à un groupe de pompage primaire (ou secondaire le cas échéant), qui comporte une arrivée et une sortie de gaz équipées de vannes d'isolement (schéma de la Fig. 1). A l'intérieur de l'enceinte à vide sont disposés deux creusets à effet joule avec leurs connexions électriques, quatre thermocouples de régulation et de contrôle des températures ainsi que trois passages étanches de translation-rotation (Figs. 2 et 3).

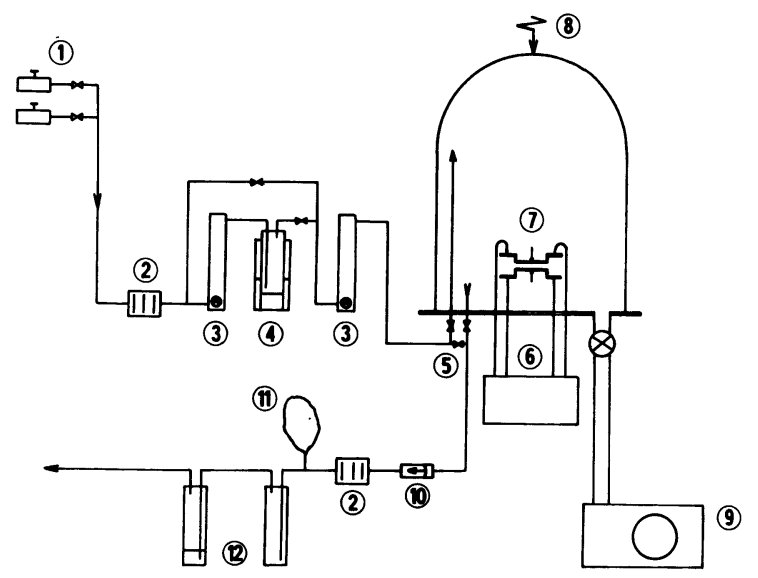

Fig. 1. - Schéma du dispositif : 1) alimentation gaz(hydrogène ou autre gaz), 2) piège azote liquide, 3) débitmètres, 4) humidificateur à saumures, 5) vannes d'isolement, 6) alimentation et régulation des creusets, 7) réacteurs d'épitaxie, 8) système de maintien de l'enceinte, 9) pompe à vide, 10) soupape anti-retour, 11) ballon, 12) visualisation du débit.

[Apparatus scheme. 1) gas supply, 2) nitrogen trap, 3) gas rate gauge, 4) humidifier, 5) cut-off taps, 6) heaters supply and regulator, 7) epitaxial reactor, 8) vessel holder, 9) vacuum pump, 10) one way valve, 11) bladder, 12) gas flow control.]

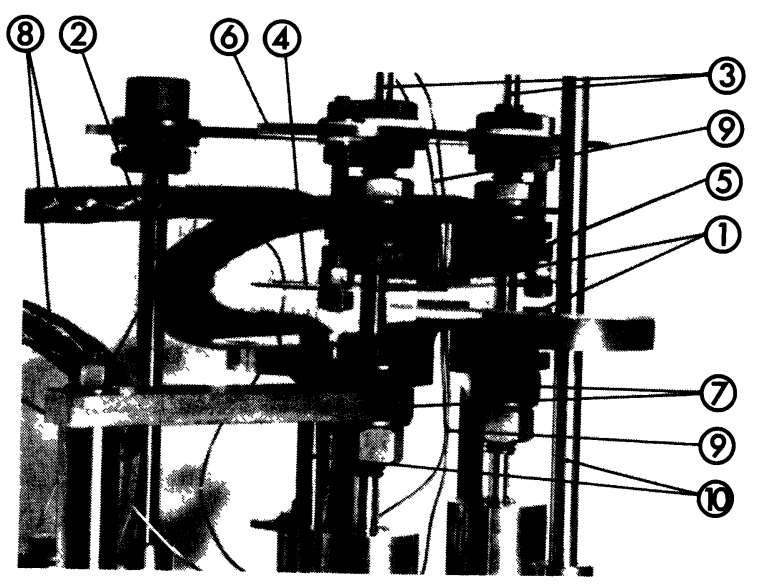

Fig. 2. - Détails du réacteur d'épitaxie.

[Epitaxial reactor.]

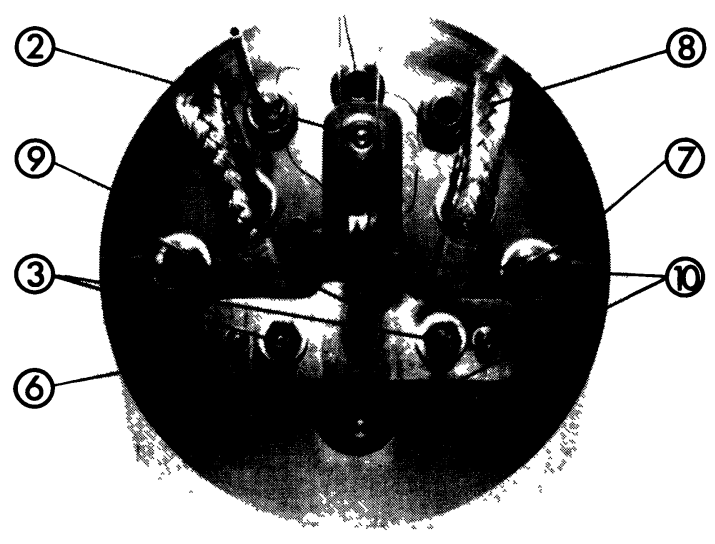

Fig. 3. - Réacteur d'épitaxie (vue de dessus).

[Epitaxial reactor (top view).]

Les creusets, en graphite de haute pureté, sont disposés l'un au-dessus de l'autre, parallèlement et à courte distance.

Le creuset-bas (1) est fixe alors que le creusethaut (1) possède un degré de translation verticale, assurée par un passage étanche (2) commandé de l'extérieur ; la translation est guidée par deux colonnes rigides (3), fixées à la platine inférieure. Associée "de manière souple au creuset-haut, une plaque d'acier inox de faible épaisseur (4) supportant une cale de silice à ouverture circulaire (5), permet de positionner la plaquette substrat et de la maintenir en contact étroit avec le creuset durant toutes les opérations. L'épaisseur de la cale de silice et le diamètre de l'ouverture circulaire fixent les dimensions de la microenceinte de croissance et constituent un des paramètres du système. La rigidité de l'ensemble est assurée par une plaque (6) située au-dessus du creuset supérieur. Des barres de connexion rigides (7) et des tresses souples (8) permettent l'alimentation électrique des creusets. A part les creusets en graphite et la cale de silice toutes les pièces du montage, y compris les conducteurs en tresses, sont en acier inoxydable. La régulation et le contrôle de la température de chaque creuset sont assurés par deux thermocouples indépendants (9) couplés à des régulateurs à angle de phase (P.I.D.).

Deux axes mobiles verticaux (10) montés sur passages étanches sont disposés symétriquement par rapport aux creusets et permettent d'intervenir de l'extérieur sur le dispositif en cours de croissance.

\section{Méthode expérimentale.}

On commence par mettre le dispositif sous vide et à dégazer les creusets en les portant à $800^{\circ} \mathrm{C}$ pendant quelques minutes. Après refroidissement, on ouvre le système et on place les échantillons sous forme de plaquettes polies, nettoyées et décapées chimiquement, de structure monocristalline pour le substrat, 
et généralement polycristalline pour la source. Plusieurs cycles de pompage-balayage d'hydrogène sont alors effectués dans l'enceinte close, puis on établit un débit précis d'hydrogène, dont la teneur en vapeur d'eau est fixée par un dispositif annexe (Fig. 1).

Pour favoriser le mode de croissance épitaxique on effectue l'opération préliminaire qui consiste à assurer un transport à partir de la source et du substrat, sur un substrat intermédiaire placé entre eux grâce à l'un des passages étanches. Ce traitement a pour effet de décaper les surfaces en révélant les plans cristallographiques favorables à la croissance. On soulève alors le creuset haut pour dégager le substrat intermédiaire, et l'on place les surfaces préparées en regard, séparées par la cale de silice. La croissance peut alors être effectuée et les conditions sont telles que le dépôt est monocristallin et épitaxié sur le substrat.

Le front de montée thermique est raide (8 secondes pour atteindre $600^{\circ} \mathrm{C}$ ) et la stabilisation permet d'assurer mieux que le degré autour de $600^{\circ} \mathrm{C}$. Il est possible ensuite d'éloigner la source pour la remplacer par une nacelle contenant un agent de dopage et d'effectuer alors un traitement de diffusion in situ.

\section{Application à la croissance de CdTe.}

Une première étude [15] avait permis de définir l'influence de la température de la source et de celle du substrat sur la vitesse de croissance d'une couche de CdTe sur un substrat du même matériau. Il a été montré en particulier que la vitesse de dépôt présentait un maximum pour une certaine température du substrat. Elle avait également révélé l'importance des effets cinétiques aux interfaces solides-vapeur, qui se traduisaient par une forte dépendance de la vitesse de croissance aux états de surface et à l'orientation cristallographique, déjà observée par ailleurs [16, 17]. L'énergie d'activation du processus a été déterminée expérimentalement [18] (Fig. 4) et se trouve être en accord avec les valeurs publiées par d'autres auteurs $[10,11,15]$. Un modèle simplifié, de portée générale, du processus de croissance a été proposé, qui permet d'atteindre les constantes thermodynamiques de la réaction de transport, même dans le cas où les espèces obtenues par la réaction ne sont pas déterminées $[19,20]$. Un autre paramètre important de la méthode, la pression et le débit du gaz réactif, a été étudié ; on a pu noter que d'une manière générale la vitesse de croissance présentait un maximum pour une certaine valeur de la pression partielle de vapeur d'eau (Fig. 5). Les vitesses de dépôt sont relativement élevées et peuvent atteindre, dans certaines conditions expérimentales, plusieurs microns par minute.

Les bonnes qualités cristallographique et électrique des couches ont permis de réaliser par cette méthode des dispositifs de conversion d'énergie solaire perfor-

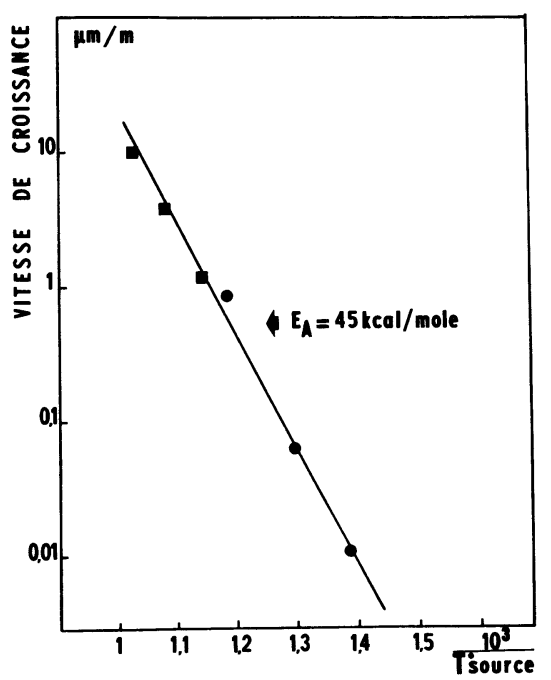

Fig. 4. - Variation de la vitesse de croissance avec l'inverse de la température absolue de la source. Les points expérimentaux sont extraits des articles référenciés [15] (๑) et [18] ( $\mathbf{a})$.

[Growth rate of CdTe versus substrate temperature. Experimental data from [15] (๑) and [18] (ם).]

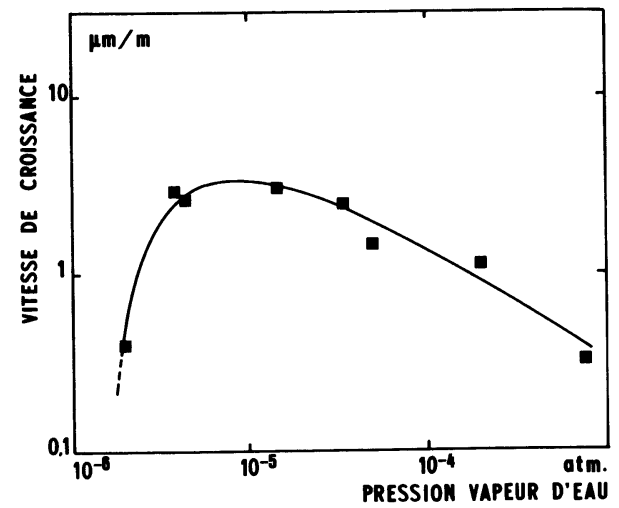

Fig. 5. - Rôle de la pression partielle de vapeur d'eau sur la vitesse de croissance de CdTe [18].

[Variation of the deposition rate of $\mathrm{CdTe}$ with the water vapour partial pressure.]

mants [21] qui exigeaient en particulier le contrôle précis de l'épaisseur de la couche (à $0,2 \mu$ près) et de la profondeur de diffusion (à $0,1 \mu$ près).

Pour conclure, la diversité des possibilités d'intervention en cours d'expérience et le contrôle aisé des différents paramètres du système font de cet appareillage un outil de recherche adapté aussi bien aux études d'épitaxie qu'à la réalisation de dispositifs électroniques.

Le même type d'appareillage a été récemment utilisé avec succès pour la croissance épitaxique de GaAs [22]. 
Bibliographie

[1] May, J. E., J. Electrochem. Soc. 112, № 7 (1965) 710-13.

[2] Lever, R. F. and Jona, F., J. Appl. Phys. 34 (1963) 3139.

[3] SiRTl, E., J. Phys. Chem. Soc. 24 (1963) 1285.

[4] Robinson, P. H., RCA Rev. 24, № 4 (1963) 574-84.

[5] Nicoll, F. H., J. Electrochem. Soc. 110, № 11 (1963) 1165.

[6] Gottlieb, G. E. and Corboy, F., RCA Rev. 24, № 4 (1963) 585-95.

[7] JoYCE, B. A., J. Cryst. Growth 3, 4 (1968) 43.

[8] Curtis, B. J. and Brunner, H., J. Cryst. Growth 6 (1970) 269.

[9] Yoshikawa, A. and Sakai, Y., J. Appl. Phys. 45, № 8 (1974) 3521.

[10] Saraie, J., Akiyama, M. and Tanaka, T., Japan. J. Appl. Phys. 11 (1972) 1758.

[11] Fahrenbruch, A. L., Vasilchenko, V., Buch, F., Mitchel, K. and Bube, R., Appl. Phys. Lett. 25 (1974) 605.

[12] Mimila-Arroyo, J., Bouazzi, A. and Cohen-Solal, 2nd Int. Symp. on CdTe, Strasbourg (1976).
[13] Lincot, D., Mimila-Arroyo, J., Triboulet, R., MarFaING, Y., Cohen-Solal, G. et Barbé, M., Int. Conf. Photov. Solar Energy Berlin (1979).

[14] Cohen-Solal, G., Lincot, D. et Barbé, M., 4th E.C. Photov. Sol. Energy Conf. Stresa, Italie (1982).

[15] Mimila-Arroyo, J., Thèse de 3e cycle, Paris VI, 1978.

[16] Shaw, D. W., Proc. 1968, Int. Symposium on GaAs, London (1969).

[17] Blakelee, A. E., Trans. Met. Soc. AIME 245 (1969) 577.

[18] Lincot, D., Thèse de 3e cycle, Paris VI, 1981.

[19] Bailly, F., Cohen-Solal, G. et Mimila-Arroyo, J., J. Electrochem. Soc. 126, № 9 (1979) 1604.

[20] Shaw, D. W., J. Electrochem. Soc. 117 (1970) 683.

[21] Barbé, M., Bailly, F., Lincot, D. et Cohen-Solal, G., IEEE Specialist. Photov. Conf. San Diego USA (1982).

[22] Mimila-ArRoyo, J. (communication personnelle). 